\title{
Mesenchymal stem cells improve intestinal integrity during severe acute pancreatitis
}

\author{
XIAO-HUANG TU, SHAO-XIONG HUANG, WEN-SHENG LI, \\ JING-XIANG SONG and XIAO-LI YANG \\ Department of General Surgery, Fuzhou General Hospital of Nanjing Military Region, \\ Fuzhou, Fujian 350025, P.R. China
}

Received November 5, 2013; Accepted May 2, 2014

DOI: $10.3892 / \mathrm{mmr} .2014 .2453$

\begin{abstract}
Severe acute pancreatitis (SAP) is an acute inflammatory disease of the pancreas that involves various distant tissues and organs. This study aimed to investigate post-tissue injury repair by mesenchymal stem cells (MSCs) in a rat model of SAP. A total of 54 pathogen-free adult male SD rats were randomly assigned to the groups SAP, SAP + MSCs and sham-operated (SO). SAP was induced by $4 \%$ sodium taurocholate, and MSCs were injected via the dorsal penile vein $1 \mathrm{~h}$ later. The amylase activity, and tumor necrosis factor (TNF)- $\alpha$ and diamine oxidase (DAO) levels were measured with an enzyme-linked immunosorbent assay (ELISA), while the expression of aquaporin (AQP)-1 was evaluated by immunohistochemical staining. The pathological score of intestinal tissues was also compared among groups. Marked improvement in intestinal necrosis, villi shedding and infiltration of inflammatory cells was observed in the SAP + MSCs group compared to the SAP and SO groups. Amylase, TNF- $\alpha$, and DAO levels were significantly increased in the SAP + MSCs group. The intestinal expression of AQP-1 was increased at 12 and $24 \mathrm{~h}$ post-MSC transplantation compared to the SO group. Rats of the SAP + MSCs group displayed higher pathological scores compared to the SAP group at all time points. Overall, these data showed that MSCs can inhibit systemic inflammation and reduce TNF- $\alpha$ release in a rat model of SAP-induced intestinal injury, suggesting that MSCs exert protective effects on the intestinal barrier during SAP.
\end{abstract}

\section{Introduction}

Severe acute pancreatitis (SAP) is an acute abdominal disease with high prevalence, severe symptoms, complicated pathogenesis and mortality as high as $20-30 \%(1,2)$. Although

Correspondence to: Dr Xiao-Huang Tu, Department of General Surgery, Fuzhou General Hospital of Nanjing Military Region, 156 West Erhuan Road, Fuzhou, Fujian 350025, P.R. China

E-mail: xiaohuangt@yeah.net

Key words: amylase, diamine oxidase, histology, mesenchymal stem cells, severe acute pancreatitis, tumor necrosis factor the mechanisms underlying SAP have not been fully elucidated, changes in secretion patterns of pancreatic acinar cells, intracellular activation of proteases and generation of inflammatory mediators may be linked to SAP pathogenesis (1). Approximately $10 \%$ of patients with acute pancreatitis show necrosis of the pancreatic and peripancreatic tissues, leading to infection of the necrotic tissue, multiple organ failure, and mortality (2). A few studies emphasized that the major damage occurring in SAP patients is not necrosis of the pancreas, but intestinal bacterial translocation, enterogenic endotoxemia and secondary pancreatic infection $(3,4)$. The small intestine may become damaged during SAP due to alterations in microcirculation associated with fluid loss, hypovolemia, splanchnic vasoconstriction and ischemia-reperfusion injury, and failure of the small intestine tends to aggravate the course of SAP (5). Intestinal permeability, which develops as a result of intestinal barrier damage early in acute pancreatitis, is directly associated with endotoxemia (6). Furthermore, increased gut permeability has been suggested to be the initial event in the bacterial contamination of pancreatic necrosis in SAP (4). Samel et al (3) focused on bacterial translocation across the gut as a functional aspect of mucosal barrier function during SAP in rats. The investigators directly observed the translocation of fluorescent bacteria from the small bowel to the pancreas, providing evidence supporting the gut origin of microorganisms responsible for the infectious complications in SAP (3). Lu et al (7) reported an increase in small intestinal capillary leakage in a rat model of SAP; they concluded that various inflammatory mediators and cytokines released during SAP may directly attack capillary endothelial cells, resulting in cell apoptosis and necrosis, and increasing intestinal barrier permeability. Although disruption of the intestinal barrier function appears to be a key step and possibly a turning point in the progression of SAP, the patients with highest mortality risk are those whose inflammatory response to pancreatic injury leads to organ failure (5). Through the action of a number of cascades, systemic inflammatory response syndrome can eventually lead to multiple organ dysfunction syndrome (8).

Mesenchymal stem cells (MSCs) isolated from various tissues, including the stroma of bone marrow and adipose tissue, have been demonstrated to exert therapeutic effects on intestinal injury as well as inflammatory, cardiovascular, degenerative and skeletal diseases (9-14). MSCs have the 
potential for proliferation and multipotent differentiation into cells of mesodermal, ectodermal and endodermal lineages (15-17). Viable strategies to foster lineage-specific differentiation of MSCs have been proposed, rendering feasible novel applications (10). MSCs possess immunoregulatory properties and have great potential for the treatment of inflammatory response; they respond to inflammation by homing to the inflamed tissues, which provides local control of inflammation and facilitates tissue repair (14). During SAP, where translocation of bacteria and toxins promotes the development of inflammation, human bone marrow-derived MSCs were shown to reduce inflammation and pancreatic tissue damage in a rat model of SAP, reducing the levels of cytokines and suppressing rat T-cell proliferation (18). Considering the unpredictable course of SAP and the absence of effective therapies, a cell-based therapeutic strategy may be promising for SAP treatment. Additional studies are needed to better understand the potential of MSCs to limit pancreatic damage in SAP, which may possibly rely on restoring the structure and function of pancreatic acinar cells (18).

In the present study, MSCs obtained by multiple digestions and passages of cells isolated from rat bone marrow were injected into male Sprague Dawley (SD) rats with taurocholate-induced SAP, in order to investigate the effects of MSC transplantation on intestinal barrier function and bacterial translocation. Our study aimed to investigate the mechanism underlying MSC-induced repair of tissue injury. Results of this study provide evidence for effective treatment of SAP with stem cell transplantation.

\section{Materials and methods}

Materials. A total of 54 specific-pathogen-free adult male SD rats, weighing $300 \pm 30 \mathrm{~g}$, were purchased from the Shanghai Laboratory Animal Co. (SLAC), Ltd. (animal license no. SCXK [Hu] 2007-0005; Shanghai, China). Animals were grown at $20-28^{\circ} \mathrm{C}$ in an environment with $40-70 \%$ humidity. The animals were allowed to accommodate to the environment for one week prior to the experiments. This study used sodium taurocholate (Sigma-Aldrich, St. Louis, MO, USA), CM-DiI (Thermo Fisher Scientific Inc., Waltham, MA, USA), enzyme-linked immunosorbent assay (ELISA) kits for detection of tumor necrosis factor (TNF)- $\alpha$ and diamine oxidase (DAO) (eBioscience Inc., San Diego, CA, USA), rabbit anti-rat monoclonal antibody targeting aquaporin (AQP)-1 (Cell Signaling Technology, Danvers, MA, USA) at a 1:2,000 dilution and biotin-conjugated rabbit anti-rat secondary polyclonal antibody at a 1:100 dilution (Cell Signaling Technology, Danvers, MA, USA). The amylase detection kit was purchased from Meikang Biotechnology (Ningbo, Jiangsu, China).

Preparation of CM-Dil-conjugated MSCs. MSCs were isolated from aseptically collected and cultured bone marrow from sacrificed male rats by multiple digestions and passages as follows: First, MSCs were digested with $0.25 \%$ trypsin (Hyclone, Logan, UT, USA) in $0.1 \%$ EDTA (Gibco-BRL, Hercules, CA, USA), and then serum containing medium was used to terminate the digestion reaction. Cells were harvested by centrifugation at $139.875 \mathrm{x} g$ for $10 \mathrm{~min}$. The supernatant was removed, and cells were washed in phosphate-buffered saline $(\mathrm{PBS})$ once. The cell suspension $\left(10^{6}\right.$ cells $\left./ \mathrm{ml}\right)$ was prepared with serum-free medium, and then CM-DiI labeling solution, a fluorescent dye that covalently conjugates to the thiol group in the cells, was added at $5 \mu \mathrm{l} / \mathrm{ml}$ of medium. The cells were resuspended and incubated at $37^{\circ} \mathrm{C}$ for $20 \mathrm{~min}$. After centrifugation at $139.875 \mathrm{x} \mathrm{g}$ for $5 \mathrm{~min}$, the supernatant was removed and cells were washed in PBS twice. Following trypan blue staining, viable cells were counted. CM-DiI-conjugated MSCs were injected into the rats via the dorsal penile vein. The rat intestine was collected, freeze-sectioned (5- $\mu \mathrm{m}$ sections) and observed by fluorescence microscopy (IX51 Biological Inverted Microscope, Olympus, Tokyo, Japan).

Establishment of the SAP rat model. Animals were deprived of food for $12 \mathrm{~h}$, but given access to water ad libitum. Following intraperitoneal anesthesia with $10 \%$ chloral hydrate at $3.0 \mathrm{ml} / \mathrm{kg}$, the rat abdomen was sterilized and a midline incision was performed at $3 \mathrm{~cm}$ from the lower part of the xiphoid. The pancreas was exposed, and the bile duct was clamped with a non-invasive clamp at the porta hepatic. Then, a 24-G trocar was inserted via the contralateral intestine, and the stylet was withdrawn. The outer cannula was inserted forward to the main pancreatic duct $(0.5-\mathrm{cm})$ and fixed. Next, $4 \%$ sodium taurocholate $(0.1 \mathrm{ml} / 100 \mathrm{~g})$ was injected by a micropump at a rate of $0.1 \mathrm{ml} / \mathrm{min}$. The cannula was left in the pancreatic duct for $5 \mathrm{~min}$ and the injection pressure was maintained. Pancreatic edema and congestion were observed, and then the clamps and cannula were removed. The intestine was sutured and returned to the abdominal cavity, followed by wound closure. After surgery, $2 \mathrm{ml}$ of normal saline were subcutaneously injected for fluid supplementation.

\section{Study design and procedures}

Animal groups. SD rats were randomly assigned to 3 groups $(\mathrm{n}=24$ each): SAP, SAP + MSCs and sham-operated (SO). Rats in the SAP and SAP + MSCs group received retrograde injection of $4 \%$ sodium taurocholate via the biliopancreatic duct to induce SAP, as described above. MSCs $\left(2 \times 10^{6}\right.$ cells $\left./ 100 \mathrm{~g}\right)$ were injected into half $(n=24)$ of the SAP-induced rats via the dorsal penile vein $1 \mathrm{~h}$ later (SAP + MSCs group). Rats in the SO group received laparotomy, and the pancreas and intestine were massaged, followed by wound closure. The SAP and SAP + MSCs group animals were sacrificed at 6, 12, 24 and $48 \mathrm{~h}$ after SAP induction. The SO group animals were sacrificed 6, 12, 24 and $48 \mathrm{~h}$ after surgery.

Detection of $\alpha$-amylase. The temporal changes in the level of amylase were measured in the 3 groups at 6, 12, 24 and $48 \mathrm{~h}$ after SAP induction. The enzymatic activity of serum amylase was detected using ethylidene-4-nitrophenyl- $\alpha-\mathrm{D}$ maltoheptaoside as a substrate, and were quantified using a spectrophotometer (7150; Hitachi Ltd., Tokyo, Japan). The amylase activity was calculated using the equation: Amylase activity $(\mathrm{U} / \mathrm{L})=\left(\mathrm{DA}_{\text {sample }} / \mathrm{min}-\mathrm{DA}_{\text {control }} / \mathrm{min}\right) * \mathrm{~F}$, where $\mathrm{F}=\mathrm{V}_{\mathrm{T}} / \mathrm{V}_{\mathrm{S}} / \mathrm{m} \varepsilon * \mathrm{P} . \mathrm{V}_{\mathrm{T}}$, total volume; $\mathrm{V}_{\mathrm{S}}$, sample volume; $\mathrm{P}=1 \mathrm{~cm}, \mathrm{~m} \varepsilon$ (molar extinction coefficient) for p-nitrophenylcarbimide $(\mathrm{pNP})=10.567$, and $\mathrm{F}=4826$ in the test.

Detection of TNF- $\alpha$ and DAO. Blood was collected from the rat abdominal aorta and the plasma was stored at $-70^{\circ} \mathrm{C}$. The TNF- $\alpha$ level and the DAP activity were detected by ELISA kits, following the manufacturer's recommendations. 

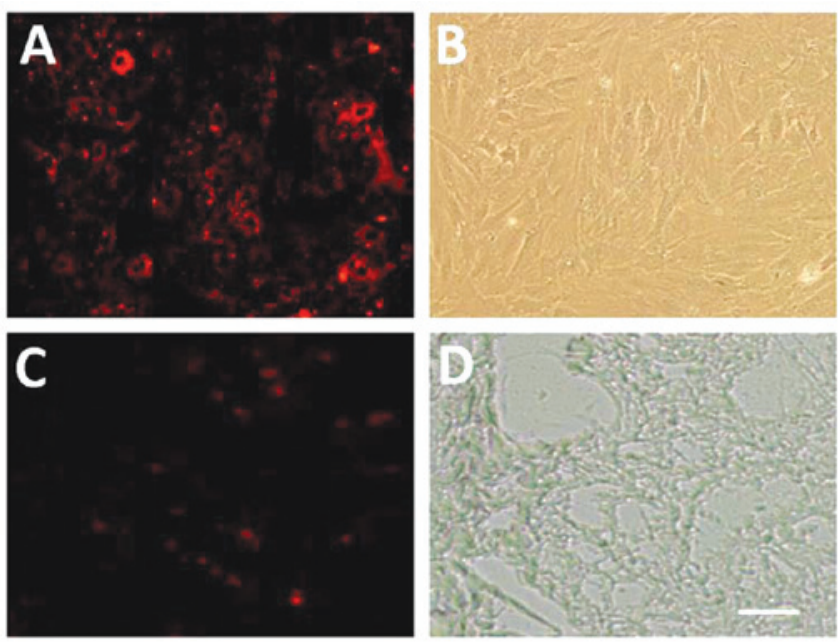

Figure 1. CM-DiI labeling and tracing of mesenchymal stem cells (MSCs). Cultured (A and B) and frozen section (C and D) of CM-DiI-labeled MSCs observed under a fluorescence microscope (A and C) and a phase contrast light microscope (B and D). Scale bar, $50 \mu \mathrm{m}$.

A

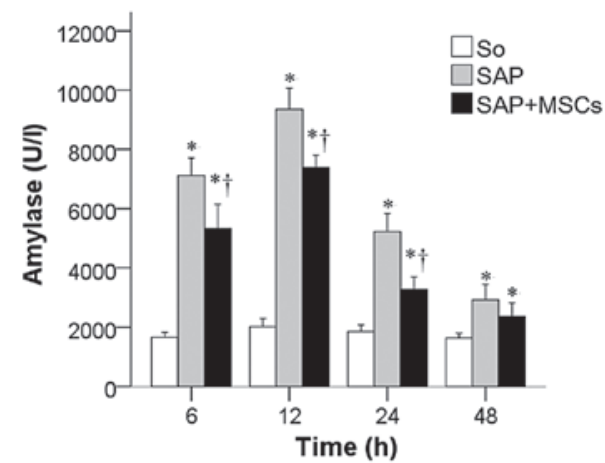

B

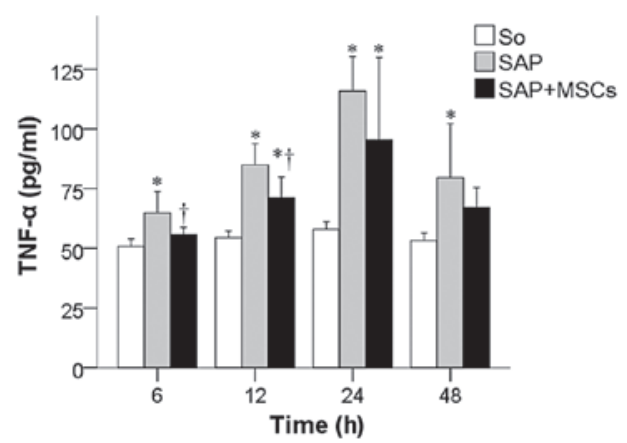

C

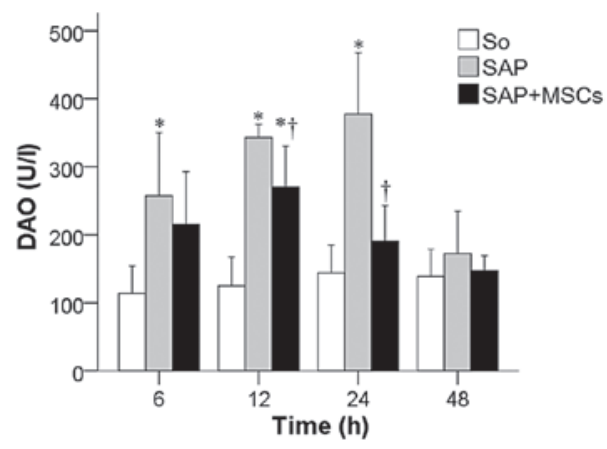

Figure 2. Temporal changes in biomarker levels in the 3 experimental groups. The levels of amylase (A), tumor necrosis factor (TNF)- $\alpha$ (B) and diamine oxidase (DAO) (C) at $6,12,24$, and $48 \mathrm{~h}$ after the induction of severe acute pancreatitis (SAP) in the SO, SAP, and SAP + MSCs groups. ${ }^{*} \mathrm{P}<0.05$ compared to the SO group; ${ }^{\dagger} \mathrm{P}<0.05$ compared to the SAP group (both after Bonferroni adjustment). Bars denote SD of the mean ( $\mathrm{n}=6$ in each group). MSCs, mesenchymal stem cells; SO, sham-operated.
Intestinal histopathology and immunohistochemical detection of AQP-1. The terminal ileum $(\sim 5 \mathrm{~cm})$ was collected and processed in paraffin-embedded sections $(4 \mu \mathrm{m})$. Then, hematoxylin and eosin (H\&E) staining was performed, and sections were observed under a light microscope (Leica DM 2000, Leica Microsystems, Wetzlar, Germany). The intestine was collected and fixed in $4 \%$ paraformaldehyde. Following dehydration, tissues were embedded in paraffin, followed by sectioning $(4 \mu \mathrm{m})$. After deparaffinization and hydration, antigen retrieval was performed in citrate acid $(\mathrm{pH} 6.0)$. Endogenous peroxidase was inactivated with $3 \% \mathrm{H}_{2} \mathrm{O}_{2}$. Following incubation with the primary anti-AQP-1 antibody at room temperature, sections were treated with biotin-conjugated rabbit anti-rat secondary antibody, followed by visualization with 3,3'-diaminobenzidine (DAB). Counterstaining was done with H\&E, followed by dehydration, transparentization and drying. After mounting in neutral gum, the sections were observed under a light microscope.

Pathological scoring of intestinal tissue. Intestinal tissue samples were fixed in $10 \%$ formaldehyde and processed in paraffin-embedded sections. Two sections were selected from each rat. H\&E staining was performed, and the sections were observed under a light microscope. For each section, 50 fields (x200) were randomly selected and scored using the scoredescribed by Chilu et al (19).

Statistical analysis. Data were expressed as mean with standard deviation (SD), and presented in bar charts. Comparisons were performed with analysis of variance (ANOVA), and resulting $\mathrm{p}$-values were adjusted for multiple testing with the Bonferroni method (20). Data were analyzed using the SPSS 15.0 statistical software (IBM, Armonk, NY, USA). P $<0.05$ were considered to indicate statistically significant differences.

\section{Results}

In vitro tracing and location of CM-DiI-labeled MSCs in the intestinal tissue. Following CM-DiI labeling, cells were observed under a fluorescence microscope. CM-DiI-labeled MSCs displayed red fluorescence. The adherent cells were 

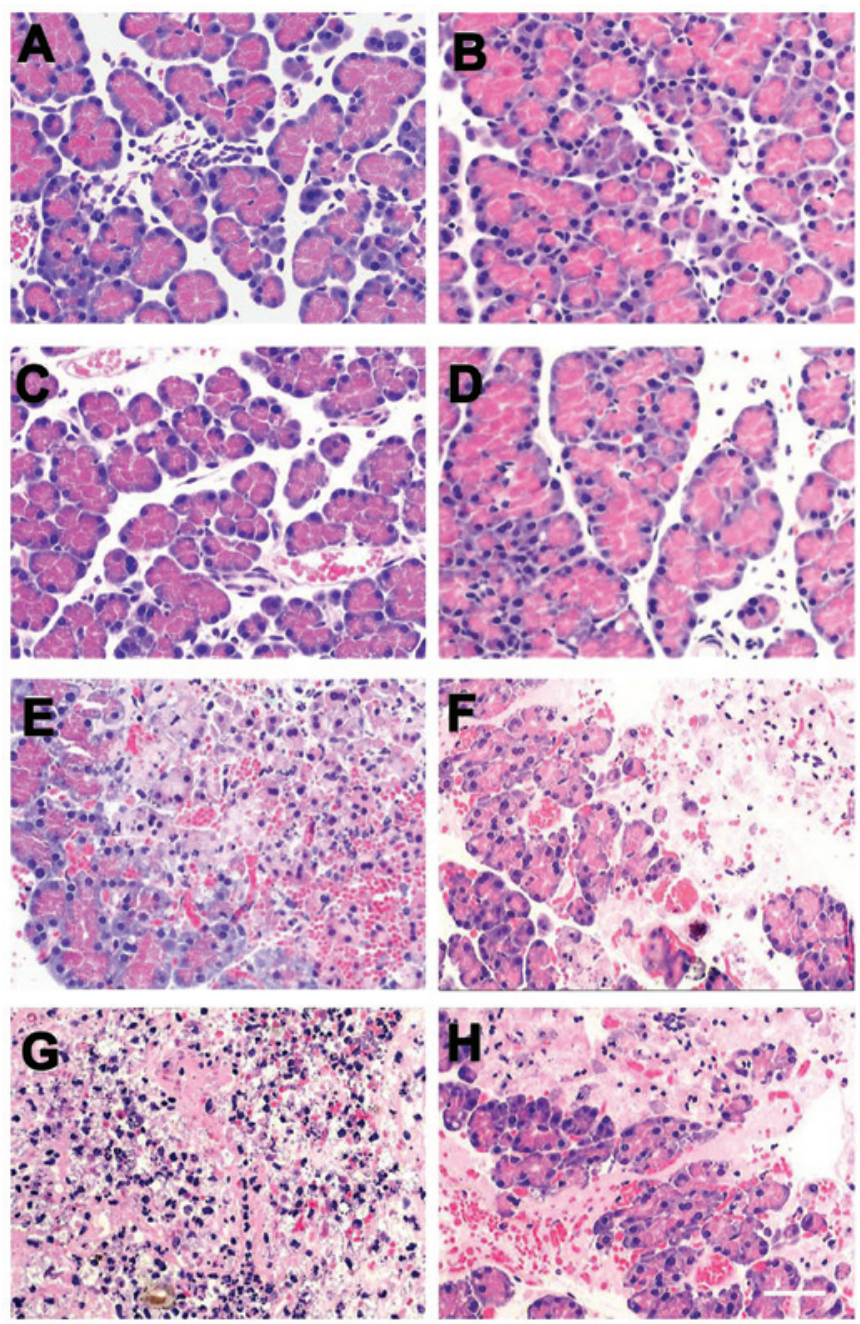

Figure 3. Temporal changes in histology of the affected pancreas Pancreatic sections stained with hematoxylin and eosin (H\&E) are from either SAP-(A, C, E and G) or SAP + MSCs-treated (B, D, F and H) rats at different time points $(6 \mathrm{~h}, \mathrm{~A}$ and $\mathrm{B} ; 12 \mathrm{~h}, \mathrm{C}$ and $\mathrm{D} ; 24 \mathrm{~h}, \mathrm{E}$ and $\mathrm{F} ; 48 \mathrm{~h}$, G and $\mathrm{H}$ ). Scale bar, $100 \mu \mathrm{m}$. SAP, severe acute pancreatitis; MSCs, mesenchymal stem cells.

spindle-like. Passage 0 MSCs were circular, with numerous granules. The fluorescence intensity was high, but not detected in the nucleus (Fig. 1A and B). Fig. 1C shows the fluorescence of frozen sections at the blue channel, revealing CM-DiIpositive cells in the intestine. Fig. 1D shows a frozen observed under a phase contrast light microscope.

Detection of markers for decreased intestinal barrier integrity. The temporal changes in the levels of intestinal barrier integrity biomarkers amylase, TNF- $\alpha$ and DAO were measured in the SAP, SAP + MSCs and SO groups at $6,12,24$, and $48 \mathrm{~h}$ after SAP induction. Amylase (Fig. 2A), TNF- $\alpha$ (Fig. 2B) and DAO values (Fig. 2C) were significantly increased in the SAP group compared to the SO group, and were decreased in the SAP + MSCs group compared to the SAP group (all $\mathrm{P}<0.05$ ).

Pathological analysis of pancreatic tissues. The structure of the pancreatic lobules as observed under the light microscope was normal. Interstitial edema and infiltration were detected
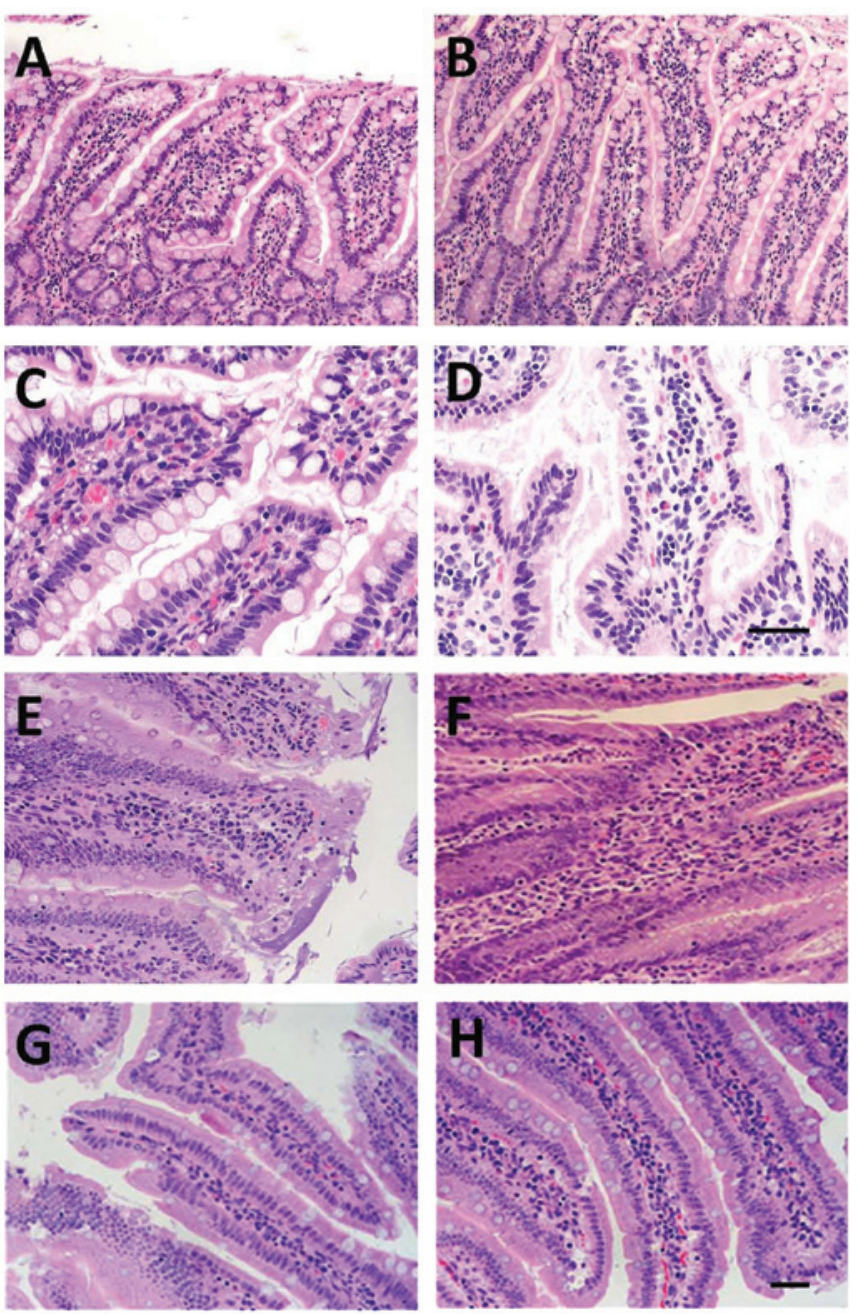

Figure 4. Temporal changes in histology of the affected intestine. Intestinal sections stained with hematoxylin and eosin (H\&E) are from either SAP(A, C, E and G) or SAP + MSCs-treated (B, D, F and H) rats at different time points ( $6 \mathrm{~h}, \mathrm{~A}$ and B; $12 \mathrm{~h}, \mathrm{C}$ and D; $24 \mathrm{~h}, \mathrm{E}$ and F; $48 \mathrm{~h}, \mathrm{G}$ and H). Scale bar, $100 \mu \mathrm{m}$ for panels A, B, E, F, G and H, and $50 \mu \mathrm{m}$ for panels $\mathrm{C}$ and D. SAP, severe acute pancreatitis; MSCs, mesenchymal stem cells.

in a few inflammatory cells, and the alveoli were normal at $6 \mathrm{~h}$ after SAP induction in the SAP group (Fig. 3A). At $12 \mathrm{~h}$ post-SAP, infiltration of inflammatory cells was detected in the pancreatic interstitial tissue, accompanied by hemorrhage, which was more severe compared to that observed $6 \mathrm{~h}$ post-SAP induction. Vacuolar degeneration was observed in the pancreatic lobules, the alveoli were largely disrupted, and hemorrhage and necrosis were detected in the adipose tissues (Fig. 3C). At $24 \mathrm{~h}$ post-SAP, massive necrosis was observed in the pancreas, blood vessels in the interstitium were markedly enlarged and hemorrhage occurred, infiltration of inflammatory cells was obvious, and alveoli were severely disrupted (Fig. 3E). At $48 \mathrm{~h}$ post-SAP, necrosis of the pancreas was aggravated, and vacuolar degeneration, congestion of blood vessels in the interstitium, infiltration of numerous inflammatory cells, patchy necrosis of adipose tissues, hemorrhage and saponification were observed (Fig. 3G). Injection of MSCs had protective effects on rats with taurocholate-induced SAP, overall reducing the damage in the pancreas (Fig. 3B, D, $\mathrm{F}$ and $\mathrm{H}$ ). 

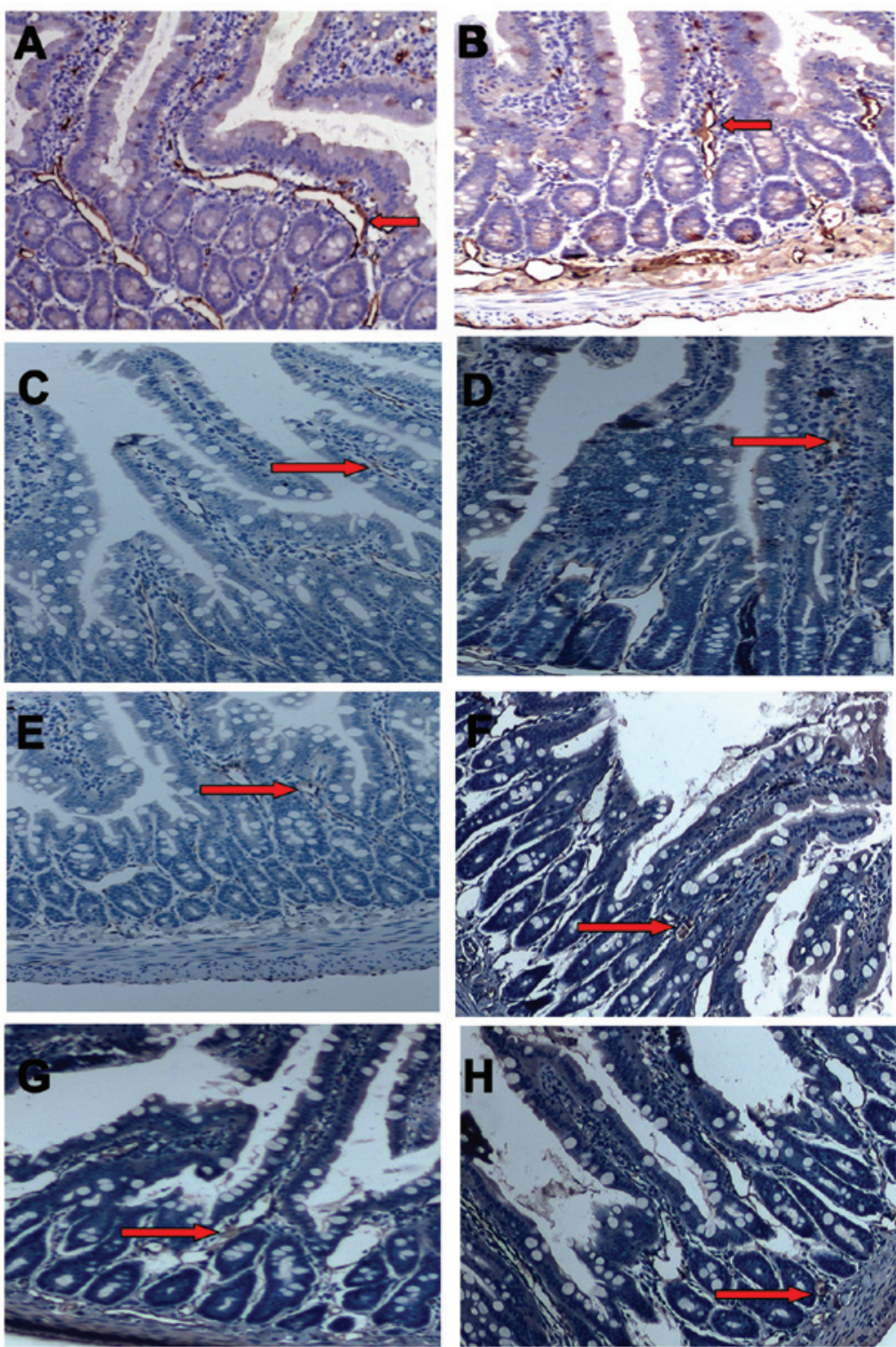

Figure 5. Immunohistochemical detection of aquaporin (AQP)-1 in affected intestines. Intestinal sections from the SO (A) and SAP (B) groups at $6 \mathrm{~h}$, and from the SAP (C, E and G) and SAP + MSCs (D, F and H) groups at three time points (12 h, C and D; $24 \mathrm{~h}, \mathrm{E}$ and F; $48 \mathrm{~h}, \mathrm{G}$ and $\mathrm{H})$ were immunostained with an antibody targeting AQP-1. Scale bar, $50 \mu \mathrm{m}$. SO, sham-operated group; SAP, severe acute pancreatitis; MSCs, mesenchymal stem cells. Red arrows, positive staining for AQP-1.

Pathological analysis of intestinal tissues. Under a light microscope, mild edema was observed in the ileal interstium, accompanied by spotty hemorrhage. In addition, the epithelium and lamina propria were mildly separated at $6 \mathrm{~h}$ post-SAP induction in the SAP group (Fig. 4A). At $12 \mathrm{~h}$, the mucosal injury of the ileum deteriorated as compared to that observed at $6 \mathrm{~h}$ post-SAP induction, and interstitial edema and infiltration of a few inflammatory cells were detected (Fig. 4C). At $24 \mathrm{~h}$ post-SAP, the villous edema of the ileum was obvious, and erosion, necrosis and shedding of intestinal villi were observed, accompanied by infiltration of numerous inflammatory cells (Fig. 4E). At $48 \mathrm{~h}$ post-SAP, the mucosal injury of the intestine was alleviated, and some repair of the intestinal mucosa was noted (Fig. 4G). At $6 \mathrm{~h}$ after treatment with MSCs, disruption at the top of the intestinal villi, interstitial edema and infiltration of neutrophils were attenuated when compared to those observed in the SAP group at corresponding time points (Fig. 4B). At $12 \mathrm{~h}$ after MSC transplantation, necrosis and shedding of the intestinal villi, and infiltration of inflammatory cells were improved compared to the SAP group at corresponding time points (Fig. 4D). At $24 \mathrm{~h}$, lodging, shortening and loosening of intestinal villi were also observed, but the extent of the damage and the infiltration of inflammation were alleviated when compared to that observed in the SAP group at corresponding time points (Fig. 4F). At $48 \mathrm{~h}$ post-MSC transplantation, pathological changes were improved in the SAP + MSCs compared to the SAP group at corresponding time points (Fig. $4 \mathrm{H}$ ).

Immunohistochemical detection of $A Q P-1$. Immunohistochemistry analysis showed that AQP-1 is highly expressed in the capillaries, small blood vessels and endothelial cells of the central chyle duct of the intestine (brown cells; Fig. 5A). At 6 h after SAP, AQP-1 expression 


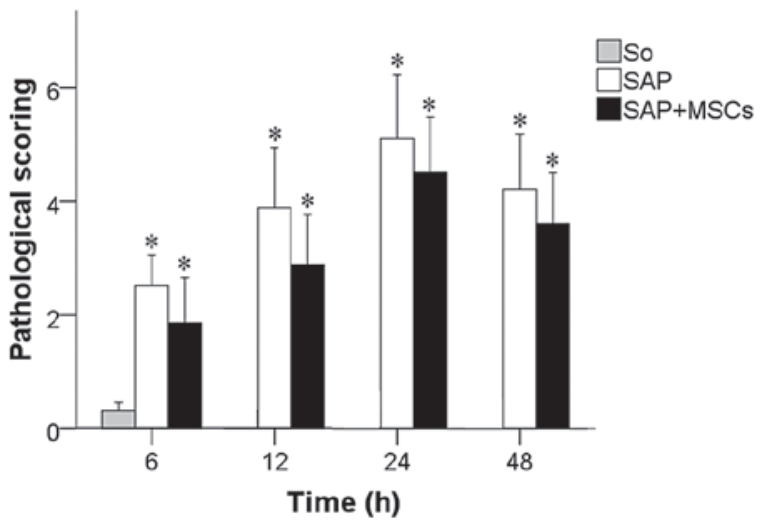

Figure 6. Pathological scoring of intestinal tissue. Hematoxylin and eosin (H\&E) staining levels in both the SAP and the SAP + MSCs groups increased continuously within 6-24 h and then decreased at $48 \mathrm{~h}$, and were significantly higher compared to the SO group at all time points. ${ }^{*} \mathrm{P}<0.05$ compared to the SO group. Bars denote SD of the mean $(n=6)$. SAP, severe acute pancreatitis; MSCs, mesenchymal stem cells; SO, sham-operated group.

was markedly reduced compared to the SO group at different time points (Fig. 5B, C, E and G), with the lowest level detected at $24 \mathrm{~h}$ after SAP induction (Fig. 5E). At $12 \mathrm{~h}$ after MSC transplantation, AQP-1 expression in the intestine was markedly increased when compared to the SAP group at different time points (Fig. 5D, F and H).

Pathological scoring of intestinal tissue. The comparison among the 3 groups with regards to the intestinal tissue pathological scores, obtained by applying the Chiu scoring method (19), is shown in Fig. 6. The H\&E staining levels in both the SAP and the SAP + MSCs group continuously increased within 6-24 h, and then decreased at $48 \mathrm{~h}$, and were significantly higher compared to the SO group at all time points $(6,12,24$ and $48 \mathrm{~h})$. Although rats of the SAP + MSCs group showed a higher staining level compared to those of the SAP group at all time points, these differences were not statistically significant (Fig. 6).

\section{Discussion}

In the present study, rats with taurocholate-induced SAP were treated with MSCs, and results demonstrated that intestinal injury was prominently improved compared to rats that were not treated with MSCs. The transplantation of MSCs reduced the levels of the intestinal enzyme amylase and the production of the inflammatory cytokine TNF- $\alpha$. In addition, the DAO level reached a peak at $12 \mathrm{~h}$ after SAP induction and then was gradually reduced, indicating that a reduced level of the enzyme was being released from mucosal epithelial cells in the injured tissue after MSC transplantation. These findings, together with those of pathological examination and microscopy, suggest that treatment with MSC contributes to the prevention or attenuation of intestinal injury during SAP, including improvement of the capillary permeability and of intestinal microcirculation. In addition, we observed that AQP-1 expression was lower in the SAP compared to the SO group starting from $6 \mathrm{~h}$ after SAP, reaching the lowest expression level at $24 \mathrm{~h}$ post-SAP induction. However, following
MSC treatment, AQP-1 expression was significantly increased in the intestine as compared to the SO group from the 12-h time point onwards. Thus, we hypothesize that MSCs may inhibit systemic inflammation and reduce TNF- $\alpha$ release, which enhances the activity of the $A Q P-1$ promoter, and upregulates mRNA and protein expression of AQP-1, leading to the attenuation of the intestinal barrier dysfunction.

Under normal conditions, the intestinal barrier is composed of mechanical, immune, biological and chemical barriers, which can potently inhibit the translocation of intestinal bacteria and toxins into extraintestinal tissues and organs, and protect from injury induced by endogenous microorganisms and toxins. However, at the early stage of SAP, the white blood cells in the pancreas and intestinal mucosa are overactivated and produce numerous inflammatory cytokines [e.g., interleukin (IL)-1, IL-6, IL-8, TNF- $\alpha$ and endotoxin (ET)] that mediate mucosal inflammation, resulting in damage of the intestinal barrier. A functional intestinal barrier does not allow endotoxins and DAO from the mucosal epithelial cells to enter the circulation. However, when the integrity of intestinal epithelial cells is altered and gut permeability increases, DAO may be released into the circulation. The translocation of intestinal bacteria (21) and endotoxemia induced by intestinal endotoxins may cause a 'second strike' $(8,22)$, resulting in secondary pancreatic infection and a cascade of inflammatory responses. Two studies by Towne et al $(23,24)$ confirmed that TNF- $\alpha$ can activate the nuclear factor (NF) $-\kappa B$ pathway via the TNF- $\alpha$ receptor, which then downregulates AQP-5 expression. AQP-1 and AQP-5 are homologous. Thus, we hypothesize that TNF- $\alpha$ can regulate AQP-1 via a similar mechanism in SAP-induced intestinal barrier dysfunction. It has been shown that AQP-1 is widely expressed in the capillaries, small blood vessels and endothelial cells of the central chyle duct in the gastrointestinal system, and that it mediates the transmembrane transport of water in the gastrointestinal tract (25). Intestinal edema manifests at the early stage of SAP. It may affect cellular viability, aggravate ischemia/hypoxiainduced injury and result in diffusion of intestinal bacteria and endotoxins into other organs and tissues, which may finally lead to systemic infection and multiple organ dysfunction (8). Thus, maintaining the intestinal barrier and preventing the translocation of bacteria and endotoxins may protect from the above-described 'second strike' following SAP.

MSCs are a group of non-hematopoietic stem cells having the potential for self-renewal and multilineage differentiation. MSCs have been used in autologous transplantation, transfection of exogenous genes and regulation of gene expression $(9,11,12,13)$. In addition, MSCs have the unique property of specifically homing to damaged tissues. This is especially valuable in SAP, since the MSCs respond to inflammation by homing to the inflamed tissues, providing local control of the inflammation and facilitating tissue repair $(11,14)$. MSCs may exert a protective effect on the intestinal barrier of animals with SAP via the following mechanisms: Numerous injury-induced cytokines in the microenvironment promote the differentiation of MSCs into specific tissues. MSCs also secrete numerous cytokines and chemokines at the injured site (25). MSCs enter the circulation at the injured sites, which improves the focal circulation and blood supply (26). This also improves the nutritional 
status, which is beneficial for the recovery of the cells and subsequently, of the tissues. We suggest that this may be one of the mechanisms underlying MSC-induced repair of injured tissues. In addition, MSCs have potent ability to regulate the immune system. Previous studies showed that MSCs can downregulate the expression of pro-inflammatory cytokines such as IL-1 and TNF- $\alpha$, and that of inducible nitric oxide synthase (iNOS), but upregulate the expression of anti-inflammatory cytokines such as IL-1 and transforming growth factor (TGF), which attenuate inflammation and improve tissue injury $(14,27)$. Jung et al (18) showed that the migration of MSCs into the inflammatory site induces the expression of forkhead box P3 (Foxp3), inhibits T cell proliferation, attenuates inflammation and facilitates tissue repair. Finally, the transplanted MSCs localize in the submucosal layer of the injured intestine and then differentiate into epithelial cells, which promotes mucosal recovery $(28,29)$. These cells can also differentiate into intestinal subepithelial myofibroblasts (ISEMFs), which may improve the microenvironment for stem cells and indirectly promote intestinal epithelial cell repair and angiogenesis in the intestine following injury (30).

Although previous studies have reported the effectiveness and safety of using bone marrow stem cells in the treatment of intestinal ischemia/reperfusion, the use of MSCs still has limitations: i) in vitro-transplanted MSCs tend to rapidly proliferate. Thus, following transplantation into animals, they may transform into malignant cells (potential tumorigenesis) (31); ii) MSCs have a large volume and potent adherent ability. The transplantation of MSCs via the tail vein usually has poor efficacy, since the majority of MSCs stay in the lung and only a few MSCs reach the injured site to exert therapeutic effects. Thus, it is difficult to optimize the number of MSCs and the frequency of transplantation; iii) although a few MSCs locate in the intestine, whether or not these cells may differentiate into intestinal epithelial cells for further repair of tissue is still unclear. These limitations in MSC transplantation may further limit the interpretation of our results.

Additional studies are needed to confirm our findings on the anti-inflammatory and immunomodulatory properties of MSCs. Our previous study (32) reported that MSCs can relieve injury of pancreatic acinar cells in a rat model of SAP, attenuate inflammation and injury in the epithelium of the small intestine, promote proliferation of the enteric epithelium and mucosal repair, thereby helping to maintain the integrity of the intestinal barrier function.

In conclusion, this study showed that SAP induces systemic inflammation in rats. Transplanted MSCs may migrate into the injured intestine, inhibiting the release of inflammatory mediators, increasing AQP-1 expression, reducing mucosal permeability of the intestine, promoting the recovery of intestinal epithelial cells and maintaining the integrity of the intestinal mucosal barrier. In the present study, transplanted MSCs inhibited systemic inflammation, reduced necrosis of intestinal epithelial cells and reduced TNF- $\alpha$ release in a rat model of SAP-induced intestinal injury, suggesting that MSCs exert a protective effect on the intestinal barrier during SAP. Our findings may provide evidence for the prevention of SAP-induced intestinal barrier dysfunction.

\section{Acknowledgements}

This study was supported by the Health and Medicine Scientific Research Foundation of Nanjing Military Area Command (no. 08Z029).

\section{References}

1. Gaisano HY and Gorelick FS: New insights into the mechanisms of pancreatitis. Gastroenterology 136: 2040-2044, 2009.

2. Warshaw AL: Improving the treatment of necrotizing pancreatitis - a step up. N Engl J Med 362: 1535-1537, 2010.

3. Samel S, Lanig S, Lux A, et al: The gut origin of bacterial pancreatic infection during acute experimental pancreatitis in rats. Pancreatology 2: 449-455, 2002.

4. Juvonen PO, Alhava EM and Takala JA: Gut permeability in patients with acute pancreatitis. Scand J Gastroenterol 35: 1314-1318, 2000

5. Capurso G, Zerboni G, Signoretti M, Valente R, Stigliano S, Piciucchi M and Delle Fave G: Role of the gut barrier in acute pancreatitis. J Clin Gastroenterol 46 (Suppl): S46-S51, 2012.

6. Koh YY, Jeon WK, Cho YK, et al: The effect of intestinal permeability and endotoxemia on the prognosis of acute pancreatitis. Gut Liver 6: 505-511, 2012.

7. Lu F, Huang H, Wang F and Chen Y: Intestinal capillary endothelial barrier changes in severe acute pancreatitis. Hepatogastroenterology 58: 1009-1017, 2011.

8. Hassoun HT, Kone BC, Mercer DW, Moody FG, Weisbrodt NW and Moore FA: Post-injury multiple organ failure: the role of the gut. Shock 15: 1-10, 2001.

9. Venkataramana NK, Kumar SK, Balaraju S, et al: Open-labeled study of unilateral autologous bone-marrow-derived mesenchymal stem cell transplantation in Parkinson's disease. Transl Res 155: 62-70, 2010.

10. Tay CY, Yu H, Pal M, et al: Micropatterned matrix directs differentiation of human mesenchymal stem cells towards myocardial lineage. Exp Cell Res 316: 1159-1168, 2010.

11. Yang YJ, Qian HY, Huang J, et al: Combined therapy with simvastatin and bone marrow-derived mesenchymal stem cells increases benefits in infarcted swine hearts. Arterioscler Thromb Vasc Biol 29: 2076-2082, 2009.

12. Abdallah BM and Kassem M: The use of mesenchymal (skeletal) stem cells for treatment of degenerative diseases: current status and future perspectives. J Cell Physiol 218: 9-12, 2009.

13. Yang F, Leung VY, Luk KD, Chan D and Cheung KM: Mesenchymal stem cells arrest intervertebral disc degeneration through chondrocytic differentiation and stimulation of endogenous cells. Mol Ther 17: 1959-1966, 2009.

14. Newman RE, Yoo D, LeRoux MA and Danilkovitch-Miagkova A: Treatment of inflammatory diseases with mesenchymal stem cells. Inflamm Allergy Drug Targets 8: 110-123, 2009.

15. Pittenger MF, Mackay AM, Beck SC, et al: Multilineage potential of adult human mesenchymal stem cells. Science 284: 143-147, 1999.

16. Pittenger MF and Martin BJ: Mesenchymal stem cells and their potential as cardiac therapeutics. Circ Res 95: 9-20, 2004.

17. Petersen BE, Bowen WC, Patrene KD, et al: Bone marrow as a potential source of hepatic oval cells. Science 284: 1168-1170, 1999.

18. Jung KH, Song SU, Yi T, et al: Human bone marrow-derived clonal mesenchymal stem cells inhibit inflammation and reduce acute pancreatitis in rats. Gastroenterology 140: 998-1008, 2011.

19. Chiu CJ, McArdle AH, Brown R, Scott H and Gurd FN: Intestinal mucosal lesion in low-flow states. I. A morphological, hemodynamic, and metabolic reappraisal. Arch Surg 101: 478-483, 1970.

20. Benjamini YD and Yekutieli D: The control of the false discovery rate in multiple testing under dependency. Ann Statist 29: $1165-1188,2001$

21. Gong Q and Li YY: The mechanism of severe acute pancreatitis combined with gastrointestinal disturbance. Int J Surg 33: 213-216, 2006 (In Chinese).

22. Ogawa M: Acute pancreatitis and cytokines: 'second attack' by septic complication lead to organ failure. Pancreas 16: 312-315, 1998.

23. Towne JE, Harrod KS, Krane CM and Menon AG: Decreased expression of aquaporin (AQP)1 and AQP5 in mouse lung after acute viral infection. Am J Respir Cell Mol Biol 22: 34-44, 2000. 
24. Towne JE, Krane CM, Bachurski CJ and Menon AG: Tumor necrosis factor-alpha inhibits aquaporin 5 expression in mouse lung epithelial cells. J Biol Chem 276: 18657-18664, 2001.

25. Brittan M, Chance V, Elia G, Poulsom R, Alison MR, MacDonald TT and Wright NA: A regenerative role for bone marrow following experimental colitis: contribution to neovasculogenesis and myofibroblasts. Gastroenterology 128: 1984-1995, 2005.

26. Chen J, Zhang ZG, Li Y, et al: Intravenous administration of human bone marrow stromal cells induces angiogenesis in the ischemic boundary zone after stroke in rats. Circ Res 92: 692-699, 2003.

27. Tögel F, Hu Z, Weiss $K$, Isaac J, Lange $C$ and Westenfelder $C$ : Administered mesenchymal stem cells protect against ischemic acute renal failure through differentiation-independent mechanisms. Am J Physiol Renal Physiol 289: F31-F42, 2005.
28. Tanaka F, Tominaga K, Ochi M, et al: Exogenous administration of mesenchymal stem cells ameliorates dextran sulfate sodiuminduced colitis via anti-inflammatory action in damaged tissue in rats. Life Sci 83: 771-779, 2008.

29. Yabana T, Arimura Y, Tanaka H, et al: Enhancing epithelial engraftment of rat mesenchymal stem cells restores epithelial barrier integrity. J Pathol 218: 350-359, 2009.

30. Gong Z and Niklason LE: Small-diameter human vessel wall engineered from bone marrow-derived mesenchymal stem cells (hMSCs). FASEB J 22: 1635-1648, 2008.

31. Zhou YF, Bosch-Marce M, Okuyama H, et al: Spontaneous transplantation of cultured mouse bone-narrow derived stromal cells. Cancer Res 66: 10849-10854, 2006.

32. Tu XH, Song JX, Xue XJ, et al: Role of bone marrow-derived mesenchymal stem cells in a rat model of severe acute pancreatitis. World J Gastroenterol 18: 2270-2279, 2012. 